\begin{tabular}{c|c|c|} 
SELECCIONES MATEMÁTICAS \\
Universidad Nacional de Trujillo \\
ISSN: $2411-1783$ (Online) \\
2020; Vol. 7(1): 29-41.
\end{tabular}

Sistemas de Cartan-Eilenberg en K-teoría Equivariante Torcida

\title{
Cartan-Eilenberg Systems in Twisted Equivariant K-Theory
}

\author{
Jesús F. Espinoza ${ }^{\circledR}$ and Rafael R. Ramos (i) \\ Received, Dec. 31, 2019 \\ Accepted, Mar. 07, 2020

How to cite this article:

Espinoza J, Ramos R. Sistemas de Cartan-Eilenberg en K-teoría Equivariante Torcida. Selecciones Matemáticas. 2020;7(1):29-41. http://dx.doi.org/10.17268/sel.mat.2020.01.04

\section{Resumen}

El objetivo de este trabajo es mostrar de manera explícita la construcción de un sistema de Cartan-Eilenberg para los grupos de K-teoría equivariante torcida sobre un $G$-complejo celular finito, con $G$ un grupo finito. Dicho sistema define una sucesión espectral cuya segunda página es dada por la cohomología de Čech asociada a una pregavilla de representaciones proyectivas de grupos de isotropía.

Palabras clave. Sistema de Cartan-Eilenberg, K-teoría equivariante torcida, sucesión espectral, complejo simplicial de Čech equivariante.

\begin{abstract}
The goal of this paper is to show explicitly the construction of a Cartan-Eilenberg system for the twisted equivariant $K$-theory groups of a finite $G-C W$ complex, with $G$ a finite group. Such a system defines a spectral sequence, whose second page is given by the Čech cohomology of a presheaf associated to projective representations of isotropy groups.
\end{abstract}

Keywords . Cartan-Eilenberg system, twisted equivariant K-thoery, spectral sequence, equivariant simplicial Čech complex.

1. Introducción. El propósito de este artículo es presentar una exposición autocontenida de K-teoría equivariante torcida y sus propiedades cohomológicas, así como los detalles de la construcción de una sucesión espectral de Segal de manera explícita. Aunque tal sucesión espectral corresponde a un caso particular de la sucesión espectral desarrollada en [16, Th. 22.4.4], así como en distintos trabajos en la dirección que el presente artículo se desarrolla: [4], [22], [10] y [11], es importante destacar que al restringirnos en el presente trabajo a complejos celulares finitos y acciones de grupos finitos, es posible hacer más transparente y explícita la construcción de la sucesión espectral, así como los morfismos involucrados en la construcción de su segunda página.

El resultado principal del presente trabajo es establecido en el Teorema 5.1, en donde la segunda página de la sucesión espectral antes mencionada es dada por la cohomología de Čech asociada a la pregavilla de grupos locales de K-teoría equivariante torcida. Como consecuencia de este teorema se deriva el Corolario 5.1, en donde se presenta una reformulación a una pregavilla de representaciones torcidas de grupos de isotropía.

2. K-teoría equivariante torcida. En esta sección introducimos la definición del funtor de K-teoría equivariante torcida, sus grupos relativos y superiores, y sus correspondientes propiedades cohomológicas. El contenido de esta sección está basado en [22] y [10], y fue presentado también en [11], ha sido incluido a fin de dar una presentación más autocontenida a este trabajo.

A lo largo de este trabajo, $G$ denotará un grupo finito y $\mathcal{H}$ un espacio de Hilbert complejo, separable y de dimensión infinita.

\footnotetext{
*Dpto. de Matemáticas, Universidad de Sonora, Hermosillo, Sonora, México (jesusfrancisco.espinoza@unison.mx).

${ }^{\dagger}$ Dpto. de Matemáticas, Universidad de Sonora, Hermosillo, Sonora, México (rramos @mat. uson.mx).
} 
2.1. Torcimientos Equivariantes. Iniciaremos con los elementos que permiten definir las versiones torcidas de la K-teoría equivariante.

Sea $P U(\mathcal{H})$ el grupo de operadores unitarios proyectivos sobre $\mathcal{H}$, el cual se define como el cociente del grupo unitario $U(\mathcal{H})$ por su centro, esto es,

$$
\begin{aligned}
P U(\mathcal{H}) & =\frac{\left\{T: \mathcal{H} \rightarrow \mathcal{H} \mid T T^{*}=T^{*} T=\operatorname{Id}\right\}}{\{T \in U(\mathcal{H}) \mid T S=S T, \text { para todo } S \in U(\mathcal{H})\}} \\
& =\frac{U(\mathcal{H})}{\{\zeta \cdot \operatorname{Id}|| \zeta \mid=1\}}=U(\mathcal{H}) / \mathbb{S}^{1}
\end{aligned}
$$

donde hemos identificado el centro de $U(\mathcal{H})$ con $\mathbb{S}^{1}$. De modo que se tiene la sucesión exacta corta

$$
1 \longrightarrow \mathbb{S}^{1} \longrightarrow U(\mathcal{H}) \stackrel{\pi}{\longrightarrow} P U(\mathcal{H}) \longrightarrow 1
$$

De hecho, si damos a $U(\mathcal{H})$ la topología fuerte (strong operator topology), entonces $U(\mathcal{H})$ tiene una estructura de haz $\mathbb{S}^{1}$-principal y localmente es el producto de $\mathbb{S}^{1}$ y $P U(\mathcal{H})$ (cf. [21, Th. 1], [9]).

Sea $\Phi: \operatorname{Hom}(G, P U(\mathcal{H})) \longrightarrow \operatorname{Ext}_{\mathrm{c}}\left(G, \mathbb{S}^{1}\right)$, la aplicación del espacio de representaciones proyectivas de $G$ al conjunto de clases de isomorfismo de extensiones $\mathbb{S}^{1}$-centrales

$$
1 \longrightarrow \mathbb{S}^{1} \longrightarrow \widetilde{G} \longrightarrow G \longrightarrow 1
$$

definida de la siguiente manera: Si $a: G \longrightarrow P U(\mathcal{H})$ es cualquier homomorfismo, éste determina una extensión $\mathbb{S}^{1}$-central de $G$ dada por $\widetilde{G}:=a^{*} U(\mathcal{H})$, como se muestra en el diagrama

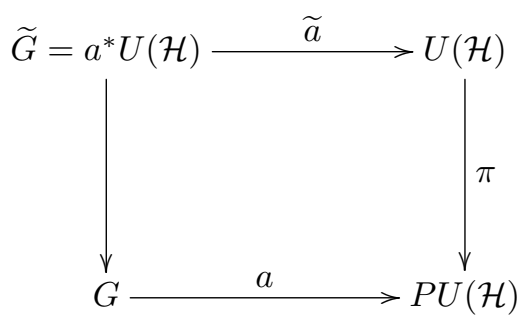

de modo que $\Phi$ es definida por

$$
(a: G \longrightarrow P U(\mathcal{H})) \stackrel{\Phi}{\longrightarrow} \widetilde{G}=a^{*} U(\mathcal{H})
$$

El hecho de que $\mathbb{S}^{1} \longrightarrow \widetilde{G} \longrightarrow G$ sea una extensión central implica que $\widetilde{G}$ es un haz fibrado $\mathbb{S}^{1}$ principal sobre $G$. Además, como $G$ es finito una extensión central $\widetilde{G}$ puede caracterizarce como el grupo $\widetilde{G} \cong G \times{ }_{\alpha} \mathbb{S}^{1}$, esto es, el conjunto $G \times \mathbb{S}^{1}$ de parejas $\left\{(g, a) \mid g \in G, a \in \mathbb{S}^{1}\right\}$ con el producto $\left(g_{1}, a_{1}\right)\left(g_{2}, a_{2}\right)=\left(g_{1} g_{2}, \alpha\left(g_{1}, g_{2}\right) a_{1} a_{2}\right)$ para un cociclo $\alpha \in \mathcal{Z}^{2}\left(G, \mathbb{S}^{1}\right)$.

Sea $V_{\text {sc }}(\widetilde{G})$ el subespacio de $L^{2}(\widetilde{G})$ generado por los vectores sobre los cuales $\mathbb{S}^{1} \subset \widetilde{G}$ actúa por multiplicación de escalares.

Un homomorfismo $a: G \longrightarrow P U(\mathcal{H})$ es llamado estable si la representación unitaria definida por $\widetilde{a}: \widetilde{G} \longrightarrow U(\mathcal{H})$ contiene cada una de las representaciones irreducibles de $\widetilde{G}$ contenidas en $V_{\text {sc }}(\widetilde{G})$, un número infinito de veces. Se denotará por

$$
\operatorname{Hom}_{\text {st }}(G, P U(\mathcal{H})) \subset \operatorname{Hom}(G, P U(\mathcal{H}))
$$

el subconjunto de tales homomorfismos estables.

Recordemos que un haz $\Gamma$-principal $G$-equivariante $p: P \longrightarrow X$ consiste en un haz $\Gamma$-principal con una $G$-acción izquierda sobre $P$ y una $G$-acción izquierda sobre $X$ (ambas acciones conmutando con las $\Gamma$-acciones derechas), tal que $p: P \longrightarrow X$ es $G$-equivariante. En la siguiente definición introducimos un tipo especial de haces $\Gamma$-principales $G$-equivariantes que serán fundamentales para la construcción de los grupos de K-teoría equivariante torcida.

Definición 2.1. Un haz $P U(\mathcal{H})$-principal $G$-equivariante $P \longrightarrow X$ sobre el $G$-espacio $X$ es llamado estable, si para todo $x \in X$ existe una vecindad $G_{x^{-i n v a r i a n t e}} U_{x}$ de $x$ y un isomorfismo de haces $G_{x^{-}}$ equivariantes

$$
\left.P\right|_{U_{x}} \cong U_{x} \times P U(\mathcal{H})
$$


de tal manera que la $G_{x}$-acción sobre el factor $P U(\mathcal{H})$ del lado derecho es mediante algún homomorfismo estable fijo $f_{x} \in \operatorname{Hom}_{\mathrm{st}}\left(G_{x}, P U(\mathcal{H})\right)$, con $G_{x}$ el subgrupo de isotropía de $G$ en $x$.

Dos haces estables $G$-equivariantes unitarios proyectivos $P$ y $P^{\prime}$ sobre $X$ se dicen ser isomorfos si existe un homeomorfismo $G$-equivariante $P \longrightarrow P^{\prime}$ de haces $P U(\mathcal{H})$-principales. Las clases de isomorfismo de haces estables $G$-equivariantes unitarios proyectivos sobre $X$ serán denotados por

$$
\operatorname{Bun}_{G}^{\mathrm{st}}(X, P U(\mathcal{H})) \text {. }
$$

Un torcimiento $G$-equivariante sobre $X$ consiste de un haz $P U(\mathcal{H})$-principal $G$-equivariante estable sobre $X$, de modo que $\operatorname{Bun}_{G}^{\text {st }}(X, P U(\mathcal{H}))$ corresponde al conjunto de clases de isomorfismo de torcimientos $G$-equivariantes sobre $X$.

Proposición 2.1. Si G es un grupo finito y $X$ es un $G$-complejo $C W$, entonces

$$
\operatorname{Bun}_{G}^{\text {st }}(X, P U(\mathcal{H})) \cong H_{G}^{3}(X ; \mathbb{Z}),
$$

donde $H_{G}^{3}(X ; \mathbb{Z}):=H^{3}\left(X \times_{G} E G ; \mathbb{Z}\right)$ representa el tercer grupo de cohomología de Borel equivariante.

Este resultado es establecido en [3]; en [5] se proporciona una demostración mediante la construcción del correspondiente espacio universal.

2.2. K-teoría equivariante torcida. La K-teoría topológica compleja fue introducida por M. F. Atiyah y F. Hirzebruch (cf. [2]) alrededor de 1959, basándose en el trabajo de A. Grothendieck y R. Bott. Fue definida en un sentido geométrico, a saber, como la construcción de Grothendieck aplicada al monoide de clases de isomorfismo de haces vectoriales complejos de rango finito. Ésta viene a ser el primer ejemplo de una teoría de cohomología generalizada, es decir, una teoría que satisface todos los axiomas de Eilenberg-Steenrod para una teoría de cohomología (cf. [8]), excepto el axioma de la dimensión. Como tal, por el Teorema de Representabilidad de Brown, es representable y de manera independiente Atiyah y Jänich probaron que un modelo para su correspondiente $\Omega$-espectro (en dimensión par) está dado por el espacio de operadores de Fredholm Fred $(\mathcal{H})$, sobre un espacio de Hilbert separable de dimensión infinita $\mathcal{H}$. Es precisamente éste el ingrediente clave para definir las versiones torcidas asociadas a la K-teoría.

Más precisamente, del Teorema de Atiyah-Jänich tenemos que la K-teoría de un complejo celular finito $X$ satisface

$$
K^{0}(X) \cong[X, \operatorname{Fred}(\mathcal{H})],
$$

equivalentemente, si $E:=X \times \operatorname{Fred}(\mathcal{H})$ es el haz trivial sobre $X$ con fibra $\operatorname{Fred}(\mathcal{H})$ y $\Gamma(X ; E)$ denota su espacio de secciones, entonces el Teorema de Atiyah-Jänich nos dice que

$$
K^{0}(X) \cong \pi_{0}(\Gamma(X ; E)) .
$$

Las "versiones torcidas" de K-teoría son obtenidas al reemplazar el haz trivial $X \times \operatorname{Fred}(\mathcal{H})$ por un haz $E$ con grupo estructural $P U(\mathcal{H})$ y cuya fibra sea $\operatorname{Fred}(\mathcal{H})$. Existen varias dificultades técnicas en esta construcción relativas a la topología que debe tener el espacio $\operatorname{Fred}(\mathcal{H})$; en [3] puede consultarse de qué manera Atiyah y Segal dan solución a estas dificultades.

Basándose en el esquema anterior, se define la K-teoría torcida de un espacio en la presencia de una acción (cf. [3, 5]).

Definición 2.2. Sean $G$ un grupo finito y $X$ un $G$-complejo celular finito. Si $P$ es un torcimiento $G$ equivariante, la $K$-teoría $G$-equivariante $P$-torcida de $X$ se define como el grupo

$$
K_{G}(X ; P):=\pi_{0}\left(\Gamma(X ; \operatorname{Fred}(P))^{G}\right),
$$

cuyos elementos corresponden a clases de homotopía de secciones G-equivariantes del haz G-equivariante de Fredholm asociado

$$
\operatorname{Fred}(P):=P \times_{P U(\mathcal{H})} \operatorname{Fred}(\mathcal{H})
$$

Es importante señalar que el modelo de $\operatorname{Fred}(\mathcal{H})$ considerado aquí ${ }^{1}$ consiste del conjunto de parejas de operadores de Fredholm $(A, B)$ tales que $A B-1$ y $B A-1$ son compactos, con la topología sobre $\operatorname{Fred}(\mathcal{H})$ inducida por el encaje

$$
(A, B) \longmapsto(A, B, A B-1, B A-1)
$$

${ }^{1}$ En [3], M. Atiyah y G. Segal denotan este espacio por $\operatorname{Fred}^{\prime}(\mathcal{H})$. 
en $\mathcal{B} \times \mathcal{B} \times \mathcal{K} \times \mathcal{K}$, con $\mathcal{B}$ el espacio de operadores acotados en $\mathcal{H}$ con la topología compacto-abierta y $\mathcal{K}$ el espacio de operadores compactos con la topología de la norma. El hecho de que $P U(\mathcal{H})$ actúa continuamente sobre $\operatorname{Fred}(\mathcal{H})$, por conjugación, y que efectivamente $\operatorname{Fred}(\mathcal{H})$ representa un espacio clasificante para la K-teoría es demostrado en [3, Prop. 3.1]. En [3, Sec. 3] se hace un análisis de lo comentado anteriormente y se establece sobre otra posible elección del modelo para representar la K-teoría torcida.

Dado el haz $P U(\mathcal{H})$-principal $G$-equivariante $P$, se define la $G$-acción sobre el haz asociado $\operatorname{Fred}(P)=$ $P \times_{P U(\mathcal{H})} \operatorname{Fred}(\mathcal{H})$ dada por

$$
g \cdot[(\lambda,(A, B))]:=[(g \lambda,(A, B))]
$$

para $g \in G, \lambda \in P$ y $(A, B) \in \operatorname{Fred}(\mathcal{H})$.

2.3. El grupo de representaciones proyectivas. Veamos el caso particular de la K-teoría $G$-equivariante torcida del espacio que consta de sólo un punto. Para ello recordaremos brevemente la definición del grupo de representaciones proyectivas. Para una exposición más detallada puede consultarse [14, Ch. III], así como el enfoque presentado en [1, Sec. 6].

Si $V$ es un espacio vectorial complejo de dimensión finita, una aplicación $\rho: G \rightarrow \operatorname{Aut}(V)$ es llamada una representación proyectiva de $G$ si existe una función $\alpha: G \times G \rightarrow \mathbb{C}^{*}$ tal que $\rho(x) \rho(y)=\alpha(x, y) \rho(x y)$, para todos $x, y \in G$ y $\rho(1)=\operatorname{Id}_{V}$.

Observemos que $\alpha$ define un cociclo sobre $G$ con valores en $\mathbb{C}^{*}$, es decir, $\alpha \in \mathcal{Z}^{2}\left(G, \mathbb{C}^{*}\right)$. Las representaciones proyectivas de $G$, están en correspondencia biyectiva con homomorfismos de $G$ al espacio proyectivo asociado a $\operatorname{Aut}(V)$.

Dos representaciones proyectivas $\rho_{1}: G \rightarrow \operatorname{Aut}\left(V_{1}\right)$ y $\rho_{2}: G \rightarrow \operatorname{Aut}\left(V_{2}\right)$ se dicen ser linealmente equivalentes si existe un isomorfismo de espacios vectoriales $f: V_{1} \rightarrow V_{2}$ tal que $\rho_{2}(g)=f \rho_{1}(g) f^{-1}$ para todo $g \in G$.

Si $\alpha$ es el cociclo correspondiente a $\rho$, decimos que $\rho$ es una $\alpha$-representación sobre el espacio $V$. Si $\rho_{i}$, con $i=1,2$, son $\alpha_{i}$-representaciones sobre $V_{i}$, entonces el hecho de que $\rho_{1}$ es linealmente equivalente a $\rho_{2}$, implica que $\alpha_{1}=\alpha_{2}$ (cf. [14, Ch. III]).

Es fácil ver que dado un cociclo fijo $\alpha$, podemos tomar la suma directa de cualesquiera dos $\alpha$-representaciones y ésta es nuevamente una $\alpha$-representación. Esta suma se extiende de manera obvia a las clases de equivalencia lineal.

Definición 2.3. Definimos $M_{\alpha}(G)$ como el monoide de clases de isomorfismos lineales de $\alpha$-representaciones de $G$, respecto a la suma directa. Denotamos por $R_{\alpha}(G)$ el grupo de Grothendieck asociado a $M_{\alpha}(G)$.

Se puede demostrar que todo cociclo $\alpha \in \mathcal{Z}^{2}\left(G, \mathbb{C}^{*}\right)$ es cohomólogo a un cociclo en $\mathcal{Z}^{2}\left(G, \mathbb{S}^{1}\right)$. Además, como $\mathbb{C}$ tiene característica 0 y el grupo aditivo $\mathbb{Q} / \mathbb{Z}$ es isomorfo al subgrupo de torsión de $\mathbb{C}^{*}$ se tiene que $H^{2}\left(G, \mathbb{C}^{*}\right) \cong H^{2}(G, \mathbb{Q} / \mathbb{Z})$ (cf. [15, Cor. 9.5]). De modo que tenemos los siguientes isomorfismos

$$
H^{2}\left(G, \mathbb{S}^{1}\right) \cong H^{2}\left(G, \mathbb{C}^{*}\right) \cong H^{2}(G, \mathbb{Q} / \mathbb{Z}) \cong H^{3}(G, \mathbb{Z}),
$$

el último isomorfismo es inducido por la sucesión exacta de $\mathbb{Z} G$-módulos (cf. [15, Prop. 1.19])

$$
0 \longrightarrow \mathbb{Z} \longrightarrow \mathbb{Q} \longrightarrow \mathbb{Q} / \mathbb{Z} \longrightarrow 0
$$

y por el hecho de que $\widetilde{H}^{*}(G, \mathbb{Q})=0$ si $G$ es finito.

Por lo anterior, usaremos clases de cohomología en $H^{2}\left(G, \mathbb{S}^{1}\right)$ donde la $G$-acción sobre los coeficientes se asume trivial.

Un elemento $\alpha \in H^{2}\left(G, \mathbb{S}^{1}\right)$ corresponde a una clase de equivalencia de extensiones centrales de grupo

$$
1 \rightarrow \mathbb{S}^{1} \rightarrow \widetilde{G}_{\alpha} \rightarrow G \rightarrow 1
$$

Como $G$ es un grupo finito, el grupo $\widetilde{G}_{\alpha}$ hereda de manera inmediata una estructura de grupo de Lie compacto, donde $\mathbb{S}^{1} \rightarrow \widetilde{G}_{\alpha}$ es la inclusión de un subgrupo cerrado. Los elementos en la extensión de grupo pueden ser representados por parejas $\left\{(g, a) \mid g \in G, a \in \mathbb{S}^{1}\right\}$ con el producto $\left(g_{1}, a_{1}\right)\left(g_{2}, a_{2}\right)=$ $\left(g_{1} g_{2}, \alpha\left(g_{1}, g_{2}\right) a_{1} a_{2}\right)$.

Observación 1. Existe una correspondencia uno a uno entre clases de isomorfismo de representaciones de $\widetilde{G}_{\alpha}$ que se restringen a multiplicación escalar sobre $\mathbb{S}^{1}$ y las clases de isomorfismo de $\alpha$ representaciones de $G$, así que podemos identificar a $R_{\alpha}(G)$ con el subgrupo de $R\left(\widetilde{G}_{\alpha}\right)$ generado por las representaciones que se restringen a multiplicación escalar sobre $\mathbb{S}^{1}$. Por otro lado, si $P$ es un torcimiento $G$-equivariante sobre $X=\{*\}$, entonces $P \cong P U(\mathcal{H})$ con una $G$-acción mediante un homomorfismo 
estable $\alpha: G \longrightarrow P U(\mathcal{H})$. De modo que si $T: \mathcal{H} \longrightarrow \mathcal{H}$ es un operador de Fredholm $\widetilde{G}$-equivariante, con $\widetilde{G} \cong G \times{ }_{\alpha} \mathbb{S}^{1}$, entonces esta $\widetilde{G}$-acción está dada por multiplicación compleja sobre el central $\mathbb{S}^{1}$, de modo que los subespacios Ker $T$ y Coker $T$ son de manera natural $\widetilde{G}$-representaciones de dimensión finita, y su diferencia es un elemento del grupo $R(\widetilde{G})$, lo cual induce el homomorfismo

$$
\begin{aligned}
& \text { ind }: K_{G}(* ; P) \longrightarrow R(\widetilde{G}) \\
& T \longmapsto \operatorname{Ker} T-\operatorname{Coker} T \text {. }
\end{aligned}
$$

Es claro que ind no es sobreyectivo, ya que ind $\left(K_{G}(* ; P)\right)$ corresponde de hecho al subgrupo de las representaciones virtuales sobre las cuales el central $\mathbb{S}^{1}$ actúa por multiplicación compleja, esto es,

$$
\text { ind }: K_{G}(* ; P) \stackrel{\cong}{\longrightarrow} R_{\alpha}(G) \subset R(\widetilde{G})
$$

2.4. Grupos relativos y superiores de K-teoría equivariante torcida. Para definir los grupos relativos de $K$-teoría equivariante torcida (cf. [5]) usaremos la construcción del espacio fibra homotópica.

Recordemos que si $\varphi: A \longrightarrow B$ es cualquier aplicación continua, entonces su fibra homotópica se define mediante el siguiente proceso. Primero definimos

$$
E_{\varphi}:=\left\{(a, f) \in A \times B^{[0,1]} \mid f(0)=\varphi(a)\right\}
$$

Tal espacio tiene la propiedad de que la aplicación $E_{\varphi} \longrightarrow B$ definida por $e v_{1}:(a, f) \longmapsto f(1)$ es una fibración (cf. [13, Prop. 4.64]), cuya fibra es denotada por $F_{\varphi}$ y es llamada la fibra homotópica de $\varphi$, la cual explícitamente está dada por

$$
\left(F_{\varphi}\right)_{b_{0}}=\left\{(a, f) \in A \times B^{[0,1]} \mid f(0)=\varphi(a), f(1)=b_{0}\right\}
$$

para algún punto base $b_{0} \in B$.

Definición 2.4. Sea $G$ un grupo finito, $(X, A)$ una $G$-pareja celular finita y $P$ un torcimiento $G$ equivariante sobre $X$. Entonces, dada la aplicación de restricción

$$
\Gamma(X ; \operatorname{Fred}(P))^{G} \underset{\text { res }}{\longrightarrow} \Gamma\left(A ; \operatorname{Fred}\left(\left.P\right|_{A}\right)\right)^{G}
$$

y $F_{\text {res }}(X, A ; P)$ la correspondiente fibra homotópica, definimos los grupos relativos de K-teoría equivariante torcida por

$$
K_{G}(X, A ; P):=\pi_{0}\left(F_{\text {res }}(X, A ; P)\right)
$$

Por otro lado, como el elemento $\operatorname{Id} \in \operatorname{Fred}(\mathcal{H})$ es fijado bajo $P U(\mathcal{H})$, éste forma una sección del haz Fred $(P)$, la cual claramente es $G$-invariante. Por lo tanto, $\Gamma(X ; \operatorname{Fred}(P))^{G}$ tiene un punto base distinguido y podemos hablar sobre grupos superiores de homotopía.

Definición 2.5. Sea $G$ un grupo finito, $(X, A)$ una $G$-pareja celular finita y $P$ un torcimiento $G$ equivariante sobre $X$. Definimos

$$
K_{G}^{-i}(X ; P):=\pi_{i}\left(\Gamma(X ; \operatorname{Fred}(P))^{G}\right)
$$

Asimismo, definimos

$$
K_{G}^{-i}(X, A ; P):=\pi_{i}\left(F_{\text {res }}(X, A ; P)\right)
$$

con $F_{\mathrm{res}}(X, A ; P)$ la fibra homotópica de la restricción

$$
\Gamma(X ; \operatorname{Fred}(P))^{G} \underset{\text { res }}{\longrightarrow} \Gamma\left(A ; \operatorname{Fred}\left(P||_{A}\right)\right)^{G} .
$$


2.5. Propiedades cohomológicas. Los grupos de K-teoría equivariante torcida satisfacen ciertas propiedades, de tal manera que le dan una estructura de teoría de cohomología generalizada en un sentido que aclararemos más adelante. La demostración de estas propiedades puede consultarse en el artículo [12], donde Freed, Hopkins y Teleman demuestran (Proposición 3.3) que la K-teoría equivariante torcida de un grupoide $X$ satisface las propiedades de una teoría de cohomología generalizada, y las propiedades presentadas en esta sección corresponden al caso particular del grupoide acción $[X / G]$ con $X$ un $G$-espacio celular finito. En [5] se presenta también una demostración detallada de cada una de tales propiedades cohomológicas en el caso de la acción propia de un grupo discreto.

Sea $\mathfrak{T} \mathfrak{w i s t}$ la categoría cuyos objetos consisten de parejas de $G$-complejos celulares $(X, A)$ con un torcimiento $G$-equivariante $P$ sobre $X$. En la categoría Twist, un morfismo $f:(X, A ; P) \longrightarrow(Y, B ; Q)$ consiste en una aplicación $G$-equivariante $f: P \longrightarrow Q$ de $P U(\mathcal{H})$-haces principales que induce una aplicación $G$-equivariante $\tilde{f}:(X, A) \longrightarrow(Y, B)$, entre parejas de $G$-complejos celulares, de tal manera que el siguiente diagrama es conmutativo

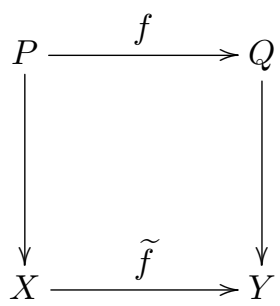

y además $P \cong \tilde{f}^{*}(Q)$.

De modo que tenemos una aplicación $G$-equivariante entre los correspondientes espacios de secciones $\Gamma(Y, B ; \operatorname{Fred}(Q)) \longrightarrow \Gamma(X, A ; \operatorname{Fred}(P))$ que desciende a un homomorfismo al nivel de los grupos de homotopía (Definición 2.5) y por lo tanto, tenemos el homomorfismo

$$
f^{*}: K_{G}^{-i}(Y, B ; Q) \longrightarrow K_{G}^{-i}(X, A ; P) .
$$

Una homotopía entre dos aplicaciones $f, g:(X, A ; P) \longrightarrow(Y, B ; Q)$ es una aplicación

$$
\left((X, A) \times[0,1] ; \pi^{*} P\right) \longrightarrow(Y, B ; Q)
$$

con $\pi: X \times[0,1] \longrightarrow X$ dada por la proyección, tal que la restricción a $(X, A) \times\{0\}$ es $f$ y la restricción a $(X, A) \times\{1\}$ es $g$.

La correspondencia

$$
K_{G}^{-i}:(X, A ; P) \longmapsto K_{G}^{-i}(X, A ; P)
$$

es un funtor homotópico contravariante de la categoría $\mathfrak{T} \mathfrak{w i s t}$ a la categoría de grupos abelianos.

El funtor de K-teoría equivariante torcida satisface las siguientes propiedades cohomológicas:

Invarianza homotópica. Si $f, g:(X, A ; P) \longrightarrow(Y, B ; Q)$, son aplicaciones homotópicas, entonces

$$
f^{*}=g^{*}: K_{G}^{-i}(Y, B ; Q) \longrightarrow K_{G}^{-i}(X, A ; P) .
$$

Sucesión exacta larga. Existe la siguiente sucesión exacta larga

$$
\begin{array}{r}
\cdots \longrightarrow K_{G}^{-i}(X, A ; P) \longrightarrow K_{G}^{-i}(X ; P) \longrightarrow K_{G}^{-i}(A ; P) \longrightarrow K_{G}^{-i+1}(X, A ; P) \longrightarrow \cdots \\
\cdots \longrightarrow K_{G}^{0}(X, A ; P) \longrightarrow K_{G}^{0}(X ; P) \longrightarrow K_{G}^{0}(A ; P)
\end{array}
$$

Aditividad. Si $(X, A)=\coprod_{\lambda}\left(X_{\lambda}, A_{\lambda}\right)$, entonces las inclusiones inducen

$$
K_{G}^{-i}(X, A ; P) \stackrel{\cong}{\longrightarrow} \prod_{\lambda} K_{G}^{-i}\left(X_{\lambda}, A_{\lambda} ; P\right) .
$$


Escisión. Si $Z \subset A$ es un $G$-subcomplejo celular contenido en el interior de $A$, entonces

$$
K_{G}^{-i}(X, A ; P) \stackrel{\cong}{\longrightarrow} K_{G}^{-i}(X \backslash Z, A \backslash Z ; P)
$$

El axioma de la dimensión para una teoría de cohomología $\mathfrak{h}^{*}$ establece que $\mathfrak{h}^{n}(*)=0$ para $n \neq 0$. Sin embargo, en el caso de los grupos de K-teoría equivariante torcida esto no se cumple, pues de hecho el Teorema de Periodicidad de Bott establece que

$$
K_{G}^{-i}(X ; P) \cong K_{G}^{-i-2}(X ; P) .
$$

Este resultado se sigue por la existencia de una equivalencia homotópica entre $\operatorname{Fred}(\mathcal{H})$ y $\Omega^{2} \operatorname{Fred}(\mathcal{H})$ la cual, al ser $U(\mathcal{H})$-equivariante (cf. [3, Sec. 4]), induce una equivalencia homotópica fibra a fibra entre $\operatorname{Fred}(P)=P \times_{P U(\mathcal{H})} \operatorname{Fred}(\mathcal{H})$ y $\Omega^{2} \operatorname{Fred}(P):=P \times_{P U(\mathcal{H})} \Omega^{2} \operatorname{Fred}(\mathcal{H})$ de la cual se sigue el isomorfismo (2.1).

Usando dicha propiedad de periodicidad para definir los K-grupos para todo entero mediante,

$$
K_{G}^{i}(X, A ; P)= \begin{cases}K_{G}^{0}(X, A ; P) & \text { si } i \text { es par } \\ K_{G}^{-1}(X, A ; P) & \text { si } i \text { es impar }\end{cases}
$$

se sigue que la K-teoría equivariante torcida es una teoría de cohomología generalizada sobre la categoría Twist.

3. Sistemas de Cartan-Eilenberg. A continuación presentaremos la construcción general de un sistema de Cartan-Eilenberg (cf. [6, Ch. XV, Sección 7] y [17, p. 58]). Mostramos también algunos ejemplos clásicos.

Sea $\mathscr{H}=\{H(p, q)\}$ una familia de módulos, uno por cada pareja $(p, q)$ en $\mathbb{Z} \cup\{-\infty, \infty\}$ tal que $-\infty \leq p \leq q \leq \infty$. La familia $\mathscr{H}$ es llamada un sistema de Cartan-Eilenberg si satisface los siguientes axiomas:

CE-1. Existen homomorfismos

$$
\eta: H\left(p^{\prime}, q^{\prime}\right) \rightarrow H(p, q)
$$

si $p \leq p^{\prime}$ y $q \leq q^{\prime}$.

CE-2. Para una terna de enteros $(p, q, r)$ tal que $-\infty \leq p \leq q \leq r \leq \infty$ existe un homomorfismo de conexión

$$
\delta: H(p, q) \rightarrow H(q, r)
$$

CE-3. La aplicación $H(p, q) \rightarrow H(p, q)$ está dada por la identidad.

CE-4. Si $p \leq p^{\prime} \leq p^{\prime \prime}$ y $q \leq q^{\prime} \leq q^{\prime \prime}$, entonces el siguiente diagrama es conmutativo:

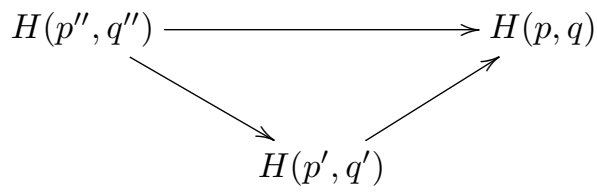

CE-5. Para $p \leq p^{\prime}, q \leq q^{\prime}$ y $r \leq r^{\prime}$, el siguiente diagrama es conmutativo:

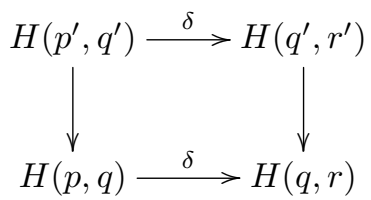

CE-6. Para $-\infty \leq p \leq q \leq r \leq \infty$, la siguiente sucesión es exacta:

$$
\cdots \longrightarrow H(q, r) \longrightarrow H(p, r) \longrightarrow H(p, q) \stackrel{\delta}{\longrightarrow} H(q, r) \longrightarrow \cdots
$$

CE-7. Para cada $q$ fijo,

$$
H(q, q) \longrightarrow H(q-1, q) \longrightarrow \cdots \longrightarrow H(p, q) \longrightarrow H(p-1, q) \longrightarrow \cdots
$$

tiene como límite directo a $H(-\infty, q)$. 
Denotamos por $H(p)$ a $H(p, \infty)$ y por $H$ a $H(-\infty, \infty)$.

Definimos

$$
\begin{aligned}
& Z_{r}^{p}=\operatorname{Im}(H(p, p+r) \rightarrow H(p, p+1)) \\
& B_{r}^{p}=\operatorname{Im}(H(p-r+1, p) \rightarrow H(p, p+1)) \\
& E_{r}^{p}=Z_{r}^{p} / B_{r}^{p} .
\end{aligned}
$$

Lo anterior define una sucesión espectral que cuando converge, lo hace a $H$.

Usualmente los módulos $H(p, q)$ tienen una graduación. En este caso asumiremos que $\eta: H\left(p^{\prime}, q^{\prime}\right) \rightarrow$ $H(p, q)$ es de grado cero, que $\delta: H(p, q) \rightarrow H(q, r)$ es de grado 1 y definimos

$$
\begin{aligned}
& Z_{r}^{p, q}=\operatorname{Im}\left(H^{p+q}(p, p+r) \rightarrow H^{p+q}(p, p+1)\right) \\
& B_{r}^{p, q}=\operatorname{Im}\left(H^{p+q}(p-r+1, p) \rightarrow H^{p+q}(p, p+1)\right) \\
& E_{r}^{p, q}=Z_{r}^{p, q} / B_{r}^{p, q} .
\end{aligned}
$$

La definición de un sistema de Cartan-Eilenberg dada anteriormente, es establecida en [17, p. 58] y se basa completamente en el esquema general dado por H. Cartan y S. Eilenberg en [6]. Tanto la definición de los módulos anteriores (graduados y bigraduados), como de los diferenciales, se encuentra desarrollada en [6, Ch. XV, Sección 1].

Ejemplo 1 (Módulos diferenciales filtrados). Todo módulo graduado $A$ con diferencial $d$ y filtración descendente $F^{p} A$, define un sistema de Cartan-Eilenberg mediante $H(p, q)=H\left(F^{p} A / F^{q} A\right)$.

Ejemplo 2. Sea $X$ un espacio topológico y $\left\{X^{p}\right\}$ una familia de subespacios definida para todo entero $p$, tal que $X^{p} \subset X^{p+1}, X^{-\infty}=\varnothing$ y $X^{\infty}=X$. Definimos

$$
H(p, q)=\bigoplus_{n} H^{n}\left(X^{q}, X^{p}\right),
$$

donde $H^{n}\left(X^{q}, X^{p}\right)$ corresponden a los grupos de cohomología de la pareja $\left(X^{q}, X^{p}\right)$ respecto a alguna teoría de cohomología fija. En este caso, la familia $\mathscr{H}=\{H(p, q)\}$ no siempre define un sistema de Cartan-Eilenberg, siendo el axioma (CE-7) el que no necesariamente se satisface. Sin embargo, en el caso de cohomología celular $H^{*}$ y complejos CW con filtración por esqueletos, dicha familia $\mathscr{H}$ define efectivamente un sistema de Cartan-Eilenberg (cf. [6, Ch. XV, Section 7]).

Ejemplo 3 (Atiyah-Hirzebruch). Si $X$ es un complejo celular finito con una filtración mediante esqueletos $\left\{X^{p}\right\}$, y $K^{n}\left(X^{q}, X^{p}\right)$ denota el $n$-ésimo grupo de K-teoría compleja de la pareja $\left(X^{q}, X^{p}\right)$, definimos

$$
H^{n}(p, q)=K^{n}\left(X^{q-1}, X^{p-1}\right) .
$$

Resulta que la familia $\mathscr{H}=\left\{H^{n}(p, q) \mid-\infty<n<\infty\right\}$ define un sistema de Cartan-Eilenberg, pues en este caso los axiomas (CE-1)-(CE-7) corresponden a las propiedades cohomológicas de la K-teoría compleja. De modo que podemos formar la sucesión espectral $E_{r}^{p, q}$, llamada la sucesión espectral de AtiyahHirzebruch (ver [2, Sec. 2]). El término $E_{1}^{p, q}$ está dado por

$$
E_{1}^{p, q}=K^{p+q}\left(X^{p}, X^{p-1}\right) \cong C^{p}\left(X, K^{q}(*)\right)
$$

siendo el diferencial $d_{1}$ el operador cofrontera ordinario; de donde se sigue que $E_{2}^{p, q} \cong H^{p}\left(X, K^{q}(*)\right) . \nabla$

De una manera completamente análoga al ejemplo anterior se puede definir el sistema de CartanEilenberg (sucesión espectral de Atiyah-Hirzebruch) para la K-teoría $G$-equivariante $P$-torcida, inducida por la filtración mediante esqueletos,

$$
E_{1}^{p, q}=K_{G}^{p+q}\left(X^{p}, X^{p-1} ; P\right),
$$

sin embargo, en este caso el término inicial no aporta ninguna ventaja computacional sobre el problema original.

En la siguiente sección se presentará una filtración del $G$-espacio $X$ asociada a una cubierta, de tal manera que la sucesión espectral inducida por el correspondiente sistema de Cartan-Eilenberg converge a la K-teoría equivariante torcida de $X$ y además, los términos iniciales son relativamente más sencillos respecto a la filtración por esqueletos; esto último se presentará en la Sección 5. 
4. La filtración asociada a una cubierta. Dado el $G$-espacio $X$ y

$$
\mathscr{U}=\left\{U_{i}\right\}_{i=0}^{\mathbf{N}}
$$

una $G$-cubierta finita por subconjuntos cerrados de $X$, construiremos un espacio asociado $W_{\mathscr{U}}$ de tal manera que $K_{G}^{*}\left(W_{\mathscr{U}} ; P\right) \cong K_{G}^{*}(X ; P)$.

La construcción de este espacio fue dada por G. Segal en [20, Sec. 5] y [19].

Sea $N_{\mathscr{U}}$ el nervio de la cubierta $\mathscr{U}$, esto es, el complejo simplicial finito cuyos simplejos son los subconjuntos de la forma $\left\{0 \leq i_{0}<i_{1}<\cdots<i_{n} \leq \mathbf{N}\right\}$ tales que

$$
U_{i_{0}} \cap U_{i_{1}} \cap \cdots \cap U_{i_{n}} \neq \varnothing
$$

y $\left|N_{\mathscr{U}}\right|$ su realización geométrica. Denotaremos por $\mathbf{J}_{(n)}$ tal $(n+1)$-tupla $\left(i_{0}, i_{1}, \ldots, i_{n}\right)$. Asimismo, usaremos la notación

$$
U_{\mathbf{J}_{(k)}}=U_{i_{0}} \cap U_{i_{1}} \cap \cdots \cap U_{i_{n}}
$$

Definimos $W_{\mathscr{U}}$ como el subespacio cerrado

$$
\bigcup_{0 \leq i \leq N} U_{\mathbf{J}_{(i)}} \times\left|\mathbf{J}_{(i)}\right|
$$

del producto $X \times\left|N_{\mathscr{U}}\right|$ y $w: W_{\mathscr{U}} \longrightarrow X$ la proyección en el primer factor. El subespacio $W_{\mathscr{U}}$ tiene una $G$-acción diagonal dada por la $G$-acción de $X$ en el primer factor y la $G$-acción trivial en el segundo factor; esta $G$-acción hace que $w: W_{\mathscr{U}} \longrightarrow X$ sea $G$-equivariante.

Por otro lado, sea $X_{p}$ el conjunto de puntos $x \in X$ que se encuentran contenidos en al menos $p+1$ elementos distintos de $\mathscr{U}$, esto es, $x \in U_{\mathbf{J}_{(p)}}$ para algún $\mathbf{J}_{(p)}$. Como la cubierta es finita, con $\mathbf{N}$ elementos, se tiene que $X_{p}=\varnothing$ para $p \geq \mathbf{N}$, esto es,

$$
X_{\mathbf{N}}=X_{\mathbf{N}+1}=X_{\mathrm{N}+2}=\cdots=\varnothing .
$$

De tal manera que tenemos una filtración de $X$ por subespacios,

$$
X=X_{0} \supset X_{1} \supset X_{2} \supset \cdots \supset X_{\mathbf{N}-1} \supset X_{\mathbf{N}}=\varnothing,
$$

la cual, mediante $w: W_{\mathscr{U}} \longrightarrow X$ induce una filtración sobre $W_{\mathscr{U}}$, a saber, $W_{p}=w^{-1}\left(X_{p}\right)$. De modo que tenemos el diagrama conmutativo,

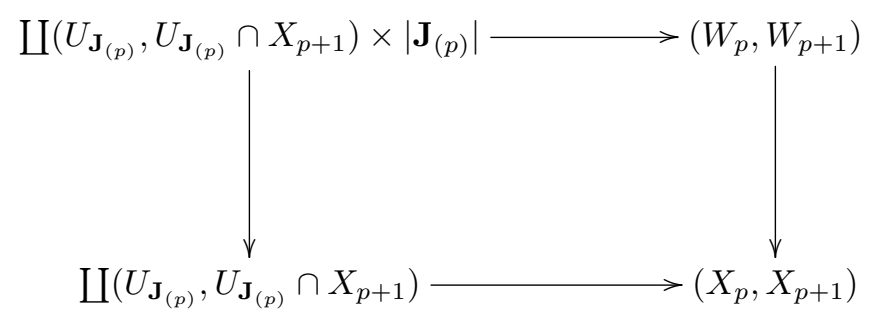

cuyas aplicaciones horizontales son dadas por homeomorfismos relativos $G$-equivariantes y la aplicación vertical de la izquierda del diagrama es una $G$-equivalencia homotópica.

De lo anterior, se sigue que

$$
K_{G}^{*}\left(W_{p}, W_{p+1} ; P\right) \cong K_{G}^{*}\left(X_{p}, X_{p+1} ; P\right)
$$

para todo $p$. Luego, como los grupos $E_{1}^{p, q}=K_{G}^{p+q}\left(W_{p}, W_{p+1} ; P\right)$ son triviales para $p \geq \mathbf{N}$ y para $p<0$, se sigue que el primer término de la sucesión espectral que éstos definen, está localizada en $\mathbf{N}$ columnas en el plano cartesiano, desde la columna $p=0$ hasta la columna $p=\mathbf{N}-1$. Luego, las diferenciales

$$
d_{r}: E_{r}^{p, q} \longrightarrow E_{r}^{p+r, q-r+1}
$$

son todas triviales para $r \geq \mathbf{N}$ y por lo tanto la sucesión espectral converge en el término $\mathbf{N}$.

Así, hemos demostrado el siguiente resultado.

Lema 4.1. $K_{G}^{*}\left(W_{\mathscr{U}} ; P\right) \cong K_{G}^{*}(X ; P)$.

Este lema es importante para la demostración del Teorema 5.1 que se presenta en la siguiente sección. 
5. Sucesión espectral para K-teoría equivariante torcida. En esta sección mostraremos cómo el sistema de Cartan-Eilenberg asociado a la K-teoría equivariante torcida del espacio $W_{\mathscr{U}}$ relativo a la $G$ cubierta de $X$ define una sucesión espectral cuya segunda página es dada por la cohomología de Čech asociada a una pregavilla de representaciones torcidas de grupos de isotropía, con ciertas restricciones sobre la cubierta que se establecerán más adelante.

La sucesión espectral que se presenta en esta sección corresponde a un caso particular de la sucesión espectral desarrollada en [4] (cf. [16]). Sin embargo, al restringirnos en el presente trabajo a complejos celulares finitos y acciones de grupos finitos, es posible hacer más transparente y explícita la construcción de la sucesión espectral, así como los morfismos involucrados en la construcción de su segunda página.

Teorema 5.1. Sea $G$ un grupo finito, $X$ un $G$-complejo celular finito y $P$ un torcimiento equivariante. Dada una $G$-cubierta finita $\mathscr{U}=\left\{U_{i}\right\}_{i=0}^{\mathbf{N}}$ de subconjuntos cerrados de $X$, existe una sucesión espectral $E_{r}^{p, q}$ tal que

$$
E_{2}^{p, q}=\check{H}^{p}\left(\mathscr{U}, \check{\mathcal{K}}^{q}\right)
$$

y convergente a $K_{G}^{p+q}(X ; P)$, donde $\check{\mathcal{K}}^{q}$ es la pregavilla $U \mapsto K_{G}^{q}(U ; P)$.

Demostración: Al espacio $W_{\mathscr{U}}$, definido en (4.2), podemos asociarle una filtración

$$
W^{0} \subset W^{1} \subset \cdots \subset W_{\mathscr{U}}
$$

dada por

$$
W^{p}:=\bigcup_{0 \leq i \leq p} U_{\mathbf{J}_{(i)}} \times\left|\mathbf{J}_{(i)}\right|
$$

esto es, la imagen inversa del $p$-esqueleto de $\left|N_{\mathscr{U}}\right|$. A esta filtración le corresponde un sistema de CartanEilenberg y en consecuencia una sucesión espectral convergiendo a $K_{G}^{*}\left(W_{\mathscr{U}} ; P\right)$ cuyo primer término está dado por $K_{G}^{p+q}\left(W^{p}, W^{p-1} ; P\right)$. Luego, a fin de reformular este primer término y poder expresarlo en forma más explícita aplicamos el funtor $K_{G}^{*}(-; P)$ a la sucesión

$$
X<\amalg U_{\mathbf{J}_{(0)}} \leftarrow \amalg U_{\mathbf{J}_{(1)}}<\coprod U_{\mathbf{J}_{(2)}} \cdots
$$

donde las flechas son dadas por inclusión y obtenemos la sucesión

$$
K_{G}^{*}(X ; P) \longrightarrow \prod K_{G}^{*}\left(U_{\mathbf{J}_{(0)}} ; P\right) \longrightarrow \prod K_{G}^{*}\left(U_{\mathbf{J}_{(1)}} ; P\right) \longrightarrow \prod K_{G}^{*}\left(U_{\mathbf{J}_{(2)}} ; P\right) \cdots
$$

donde los morfismos satisfacen las condiciones de pegado inducidas por las funciones de pegado del torcimiento $G$-equivariante $P$. Más específicamente, como $P$ es un haz $P U(\mathcal{H})$-principal se sigue que la función de transición sobre $U_{i} \cap U_{j}$ de $\left.P\right|_{U_{i}}$ a $\left.P\right|_{U_{j}}$ define un haz de línea $L_{i j}$ sobre $U_{i} \cap U_{j}$, de modo que se tiene el isomorfismo

$$
\left.\left.L_{i j} \otimes P\right|_{U_{i}} \longrightarrow P\right|_{U_{j}}
$$

sobre $U_{i} \cap U_{j}$, de tal manera que sobre cada triple intersección tenemos que se cumple la condición de cociclo dada por el isomorfismo

$$
L_{j k} \otimes L_{i j} \longrightarrow L_{i k}
$$

Esto implica que los morfismos en la sucesión son dados por la composición de restricciones y automorfismos de la K-teoría equivariante torcida.

Definimos

$$
E_{1}^{p, q}=K_{G}^{q}\left(A_{p} ; P\right), \text { donde } A_{p}:=\coprod U_{\mathbf{J}_{(p)}} .
$$

Luego, si $\Delta^{p}$ es el $p$-simplejo estándar y $\partial \Delta^{p}$ su $(p-1)$-esqueleto, de la terna $\left(A_{p} \times \Delta^{p}, A_{p} \times \partial \Delta^{p}, A_{p} \times\right.$ $\Delta^{p-1}$ ) obtenemos la sucesión exacta

$$
0 \longrightarrow K_{G}^{p+q-1}\left(A_{p} \times \partial \Delta^{p}, A_{p} \times \Delta^{p-1} ; P\right) \longrightarrow K_{G}^{p+q}\left(A_{p} \times \Delta^{p}, A_{p} \times \partial \Delta^{p} ; P\right) \longrightarrow 0,
$$

esto es, un isomorfismo

$$
K_{G}^{p+q}\left(A_{p} \times \Delta^{p}, A_{p} \times \partial \Delta^{p} ; P\right) \cong K_{G}^{p+q-1}\left(A_{p} \times \partial \Delta^{p}, A_{p} \times \Delta^{p-1} ; P\right),
$$


y usando el principio de escisión en la pareja $\left(A_{p} \times \partial \Delta^{p}, A_{p} \times \Delta^{p-1}\right)$, tenemos

$$
K_{G}^{p+q-1}\left(A_{p} \times \partial \Delta^{p}, A_{p} \times \Delta^{p-1} ; P\right) \cong K_{G}^{p+q-1}\left(A_{p} \times \Delta^{p-1}, A_{p} \times \partial \Delta^{p-1} ; P\right)
$$

en consecuencia

$$
K_{G}^{p+q}\left(A_{p} \times \Delta^{p}, A_{p} \times \partial \Delta^{p} ; P\right) \cong K_{G}^{p+q-1}\left(A_{p} \times \Delta^{p-1}, A_{p} \times \partial \Delta^{p-1} ; P\right)
$$

e inductivamente, se sigue que

$$
E_{1}^{p, q} \cong K_{G}^{p+q}\left(A_{p} \times \Delta^{p}, A_{p} \times \partial \Delta^{p} ; P\right) .
$$

Luego, de las propiedades cohomológicas de la K-teoría equivariante torcida y la equivalencia homotópica relativa $\left(A_{p} \times \Delta^{p}, A_{p} \times \partial \Delta^{p}\right) \longrightarrow\left(W^{p}, W^{p-1}\right)$, se sigue que,

$$
E_{1}^{p, q} \cong K_{G}^{p+q}\left(W^{p}, W^{p-1} ; P\right) .
$$

Por lo tanto, de la conmutatividad del diagrama

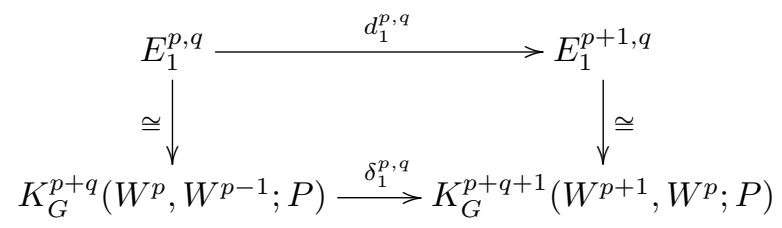

obtenemos que la sucesión espectral $E_{r}^{p, q}$ converge a $K_{G}^{*}\left(W_{\mathscr{U}} ; P\right)$.

Finalmente, si $\check{\mathcal{K}}^{q}$ es la pregavilla $U \mapsto K_{G}^{q}(U ; P)$, entonces por definición se sigue que

$$
E_{2}^{p, q} \cong \check{H}^{p}\left(\mathscr{U}, \check{\mathcal{K}}^{q}\right)
$$

Lo cual concluye la demostración del teorema.

Tomando el límite directo de la familia de sucesiones espectrales correspondientes a cada cubierta cerrada e indexada por éstas, del Teorema 5.1 obtenemos que existe una sucesión espectral $E_{r}^{p, q}$ tal que

$$
E_{2}^{p, q}=H^{p}\left(X, \mathcal{K}^{q}\right)
$$

y convergente a $K_{G}^{p+q}(X ; P)$, donde $\mathcal{K}^{q}$ es la gavilla asociada a la pregavilla $U \mapsto K_{G}^{q}(U ; P)$.

Por otro lado, como $G$ es finito y $X$ es un $G$-CW-complejo finito se sigue que $X$ es un $G$-ANR (cf. [18, Sec. 3]) y por lo tanto, podemos tomar una cubierta cerrada equivariantemente contraíble $\mathscr{U}$ y cuyas intersecciones arbitrarias no vacías sean también $G$-contraíbles, entonces para cada cerrado $U_{i} \in \mathscr{U}$ se tiene

$$
\lambda_{i}: K_{G}\left(U_{i} ; P\right) \stackrel{\cong}{\longrightarrow} K_{G}(* ; P) \cong R_{\alpha}\left(G_{i}\right)
$$

con $G_{i}$ el subgrupo de isotropía de $U_{i}$ y $\alpha$ el cociclo asociado al haz $P$ (ver Observación 1); en una doble intersección $U_{i} \cap U_{j}$,

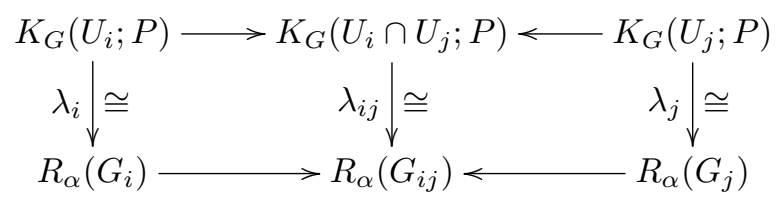

de modo que si los morfismos en el renglón inferior son tomados de tal manera que hacen conmutar cada diagrama, entonces cada morfismo $\psi_{i}: R_{\alpha}\left(G_{i}\right) \longrightarrow R_{\alpha}\left(G_{i j}\right)$ está dado explícitamente como

$$
\psi_{i}=\rho_{i j} \cdot \text { res }
$$

con $\rho_{i j} \in H_{G}^{3}\left(U_{i} \cap U_{j} ; \mathbb{Z}\right) \cong H^{2}\left(G ; \mathbb{S}^{1}\right)$, res : $R_{\alpha}\left(G_{i}\right) \longrightarrow R_{\alpha}\left(G_{i j}\right)$ la restricción y los automorfismos $\rho_{i j}$ satisfaciendo la correspondiente condición de cociclo sobre las triples intersecciones. Sobre las intersecciones múltiples se cumplen las propiedades análogas. 
De lo anterior, tenemos una familia de isomorfismos (flechas verticales), compatibles con los morfismos de restricción,

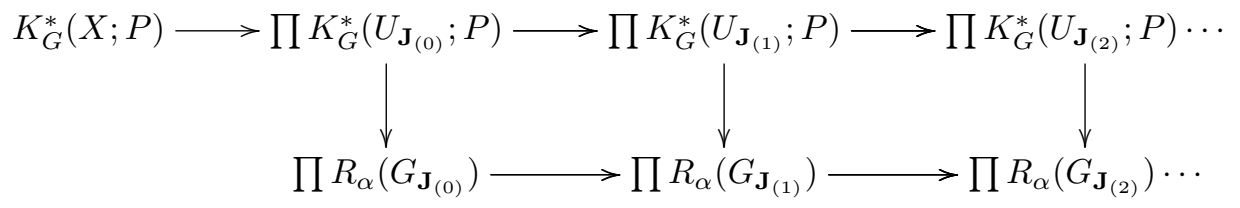

de tal manera que las correspondientes cohomologías de Čech son isomorfas y en consecuencia la sucesión espectral inducida por esta sucesión converge a $K_{G}^{p+q}(X ; P)$ y por lo tanto tenemos el siguiente resultado.

Corolario 5.1. Sea $G$ un grupo finito, $X$ un $G$-complejo celular finito y $P$ un torcimiento $G$-equivariante sobre $X$. Entonces existe una sucesión espectral $E_{r}^{p, q}$ tal que

$$
E_{2}^{p, q}= \begin{cases}\check{H}^{p}\left(\mathscr{U}, R_{\alpha}(-)\right) & \text { si q es par } \\ 0 & \text { si q es impar }\end{cases}
$$

y convergente a $K_{G}^{p+q}(X ; P)$, donde $\alpha$ es el elemento en $H_{G}^{3}(* ; \mathbb{Z}) \cong H^{2}\left(G ; \mathbb{S}^{1}\right)$ correspondiente a la imagen de $P$ mediante el homomorfismo $H_{G}^{3}(X ; \mathbb{Z}) \longrightarrow H_{G}^{3}(* ; \mathbb{Z})$ y $R_{\alpha}(-)$ es el sistema de coeficientes definido por $R_{\alpha}: G / H \longmapsto R_{\alpha}(H)$.

6. Ejemplo. Sea $G$ un grupo finito que actúa trivialmente sobre la esfera de dimensión par $S^{2 n}$ y sea $P$ un torcimiento $G$-equivariante sobre $S^{2 n}$.

Se tiene que el tercer grupo de cohomología de Borel $G$-equivariante de $S^{2 n}$ presenta la siguiente descomposición de Künneth,

$$
H_{G}^{3}\left(S^{2 n} ; \mathbb{Z}\right) \cong H^{0}\left(S^{2 n} ; \mathbb{Z}\right) \otimes H^{3}(B G ; \mathbb{Z}) \bigoplus H^{2}\left(S^{2 n} ; \mathbb{Z}\right) \otimes H^{1}(B G ; \mathbb{Z})
$$

Luego, $H^{1}(B G ; \mathbb{Z})=0$ ya que $G$ es finito y por lo tanto $H_{G}^{3}\left(S^{2 n} ; \mathbb{Z}\right) \cong H^{3}(B G ; \mathbb{Z})$ consiste sólo de elementos de torsión discreta. De modo que

$$
K_{G}^{*}\left(S^{2 n} ; P\right) \cong K^{*}\left(S^{2 n}\right) \otimes R_{\alpha}(G)
$$

donde $\alpha \in H^{3}(B G ; \mathbb{Z})$ representa la clase en cohomología del torcimiento equivariante $P$. El isomorfismo (6.1) se sigue de que en este caso el grupo de K-teoría equivariante torcida $K_{G}^{*}\left(S^{2 n} ; P\right)$ es isomorfo al grupo de K-teoría equivariante con torsión discreta (ver [1] y [7]) y tal isomorfismo es establecido en [1, Lemma 7]. Por otro lado, del isomorfismo anterior y [7, Th. 4.4], la sucesión espectral inducida por el sistema de Cartan-Eilenberg tiene como segunda página el siguiente término,

$$
E_{2}^{p, q} \cong H^{p}\left(S^{2 n} ; \mathbb{Z}\right) \otimes R_{\alpha}(G) \Longrightarrow K^{p+q}\left(S^{2 n}\right) \otimes R_{\alpha}(G),
$$

para $q$ par y, $E_{2}^{p, q}=0$ para $q$ impar. Luego, del Teorema de Periodicidad de Bott para K-teoría, se sigue que $E_{2}^{p, q} \Longrightarrow K^{p}\left(S^{2 n}\right) \otimes R_{\alpha}(G)$ para $q$ par. Este resultado es consistente con los resultados obtenidos en [1] y [7].

El argumento anterior es válido, de hecho, para cualquier esfera $G$-trivial $S^{m}$, con $G$ finito, de dimensión $m>3$. Los casos $m=1$ y $m=3$ presentan algunas diferencias interesantes y en [4] se presentan algunos resultados, algunos de los cuales son consecuencia directa de las propiedades cohomológicas de la K-teoría equivariante torcida.

Agradecimientos. El autor Jesús F. Espinoza agradece el apoyo de PRODEP y de la Universidad de Sonora en la realización de este trabajo.

ORCID and License

Jesús F. Espinoza https://orcid.org/0000-0001-5601-3634

Rafael R. Ramos https://orcid.org/0000-0002-6406-0083

This work is licensed under the Creative Commons Attribution-NoComercial-ShareAlike 4.0. 


\section{Referencias}

[1] Adem A, Ruan Y. Twisted Orbifold K-Theory. Comm. Math. Phys. 2003; 237(3): 533-556.

[2] Atiyah M, Hirzebruch F. Vector bundles and homogeneous spaces. Amer. Math. Soc. Symp. in Pure Math. 1961; 3: 7-38.

[3] Atiyah M, Segal G. Twisted K-Theory. Ukr. Mat. Visn. 2004; 1(3): 287-330.

[4] Bárcenas N, Espinoza J, Uribe B, Velázquez M. Segal's spectral sequence in twisted equivariant K-theory for proper and discrete actions. Proceedings of the Edinburgh Mathematical Society. 2018; 61(1): 121-150. https://doi.org/10.1017/S0013091517000281

[5] Bárcenas N, Espinoza J, Joachim M, Uribe B. Universal twist in equivariant $K$-theory for proper and discrete actions. Proceedings of the London Mathematical Society. 2014; 108(5): 1313-1350. https://doi.org/10.1112/plms/pdt061

[6] Cartan H, Eilenberg S. Homological algebra. Princeton Landmarks in Mathematics, 1956.

[7] Dwyer C. Twisted equivariant K-theory for proper actions of discrete groups. K-Theory. 2008; 38: 95-111.

[8] Eilenberg S, Steenrod N. Foundations of algebraic topology. Princeton University Press, 1952.

[9] Espinoza J, Uribe B. Topological properties of the unitary group. JP Journal of Geometry and Topology. 2014; 16(1): $45-55$.

[10] Espinoza J. K-Teoría Equivariante Torcida. PhD thesis, UNAM, 2013.

[11] Espinoza J. K-teoría equivariante torcida. Memorias de la XXI Semana de Investigación y Docencia en Matemáticas. Universidad de Sonora, 2011.

[12] Freed D, Hopkins J, Teleman C. Loop groups and twisted K-theory I. Journal of Topology. 2011; 4(4): 737-798.

[13] Hatcher A. Algebraic Topology. Cambridge, New York, NY., 2002.

[14] Karpilovsky G. Projective Representations of Finite Groups. Monographs in Pure and Applied Mathematics 94. Marcel Dekker, Inc., New York and Basel, 1985.

[15] Karpilovsky G. Group Representations, vol. 2 of North-Holland Mathematics Studies 177. Elsevier Science Publishers, Amsterdam, The Netherlands, 1993.

[16] May J, Sigurdsson J. Parametrized Homotopy Theory. Mathematical surveys and monographs, American Mathematical Society, 2006.

[17] McCleary J. User's Guide to Spectral Sequences. Wilmington, Delaware (U.S.A.), 1985.

[18] Schultz R. Homotopy decompositions of equivariant function spaces. II. Math. Z. 1973; 132: 69-90.

[19] Segal G. Classifying spaces and spectral sequences. Publications Mathematiques de Inst. Hautes Études Sci. Publ. Math. 1968; 34: $105-112$

[20] Segal G. Equivariant K-Theory. Publications Mathematiques de Inst. Hautes Études Sci. Publ. Math. 1968; 34: 129-151.

[21] Simms D. Topological aspects of the projective unitary group. Proc. Camb. Phil. Soc. 1970; 68: 57-60.

[22] Uribe B. Group actions on DG-Manifolds and Exact Courant Algebroids. Communications in Mathematical Physics. 2013; 318(1): 35-67. https://doi.org/10.1007/s00220-013-1669-2 